\title{
Evaluation of the Effect of Oral Zinc Sulfate on Burning Mouth Syndrome: A Double Blind Randomized Clinical Trial
}

\author{
Fateme Arbabi-Kalati, ${ }^{1,2, *}$ and Bahram Tahmtan ${ }^{1}$ \\ ${ }^{1}$ Oral Medicine Department, Zahedan University of Medical Sciences, Zahedan, IR Iran \\ ${ }^{2}$ Oral and Dental Disease Research Center, Zahedan University of Medical Sciences, Zahedan, IR Iran \\ "Corresponding author: Fateme Arbabi-Kalati, Oral Medicine Department, Zahedan University of Medical Sciences, Zahedan, IR Iran. E-mail: arbabi@zaums.ac.ir
}

Received 2015 November 03; Revised 2015 November 10; Accepted 2015 November 15.

\begin{abstract}
Background: Burning Mouth Syndrome (BMS) is a common condition that affects menopausal women and patients experience a severe burning sensation in their mouth. So far there have been no definitive treatment for this disease.

Objectives: The present study was undertaken to evaluate the efficacy of zinc supplement in improving the symptoms of burning mouth syndrome.

Patients and Methods: Sixty patients were enrolled in this study; they were randomly divided to two groups. Patients in the intervention group received replacement therapy twice daily, which continued for three months. In the control group, placebo capsules, which were completely similar to zinc sulfate capsules, were administered two times daily.

Results: Burning sensation severity in the two groups after the intervention was significantly different $(\mathrm{P}=0.001)$.

Conclusions: It can be concluded that zinc supplement might decrease the intensity of burning mouth syndrome in patients with zinc deficiency.
\end{abstract}

Keywords: Zinc, Burning Mouth Syndrome, Pain

\section{Background}

Burning Mouth Syndrome (BMS) is defined as a feeling of pain and burning in the normal oral mucosa, especially on the tongue, which affects other mucosal surfaces, including the lips, buccal mucosa and the floor of the mouth (1).

The tongue appears to be the most common location for burning sensation in the oral cavity. The symptoms usually include a burning sensation, occasional pain, disturbances in taste and xerostomia. A complaint of burning sensation is mostly reported in postmenopausal women (2).

The majority of patients with BMS are postmenopausal women over 60 years of age. The patients usually report xerostomia and loss of or changes in their taste feeling. The tip of the tongue is the most commonly affected area but other areas, including the lips, gingiva and palate, are affected, as well. The BMS etiology is considered multifactorial, including various local and systemic factors. Due to a high turn-over rate of mucous cells and trauma, these areas are sensitive to nutritional deficiencies, including minerals and vitamins. Zinc is the second most important element of the body after iron, with an important role in growth and development. Although zinc deficiency in the immune system has been reported to be related to BMS, the beneficial effects of zinc replacement therapy have not been evaluated in patients with BMS. In a study in 2010 by Cho, $26.8 \%$ of patients with BMS exhibited zinc deficiency and zinc replacement therapy experienced a decrease in burning sensation (3).

A burning sensation in the oral mucosa might occur in other oral diseases such as lichen planus and geographic tongue; however, patients with BMS are a separate group in which clinical examinations reveal normal oral mucosa, with persistent burning sensation, compared to the disease entities mentioned above (4). Burning mouth syndrome is associated with nutritional deficiencies, including vitamin $B_{1}, B_{2}, B_{6}$ and $B_{12}$ deficiencies. However, recent studies have shown that zinc deficiency can also lead to BMS. Current diagnostic criteria include continuous everyday pain in the oral cavity in association with normal oral mucosa after excluding other local and systemic diseases (5). Metabolic endocrine and nutritional disorders might give rise to BMS. Some nutritional disorders include deficiencies in iron, zinc and vitamin $B$ group, especially vita$\min B_{12}(6,7)$.

The initial diagnosis of BMS is based on the exclusion of etiologic factors. When systemic, local and psychological factors are present, treatment or their elimination usually results in significant clinical recovery from BMS. It ap- 
pears in some patients with BMS with deficiencies mentioned previously, eliminations of systemic, local and psychological factors has results in alleviation of or decrease in BMS symptoms (8).

\section{Objectives}

Given the lack of sufficient studies and data on the effect of zinc replacement therapy regarding the decrease in the severity of BMS symptoms, the present study was undertaken to evaluate the effect of zinc replacement therapy on BMS symptoms.

\section{Patients and Methods}

The present clinical trial was approved by the ethics committee of Zahedan University of Medical Sciences and registered at the Iranian center for clinical trials under the code IRCT20131209313N5 and was available at www.irct.ir during years 2013 to 2014 .

Patients with BMS referring to Zahedan faculty of dentistry, who had zinc deficiency in laboratory evaluations, were included in the study. For excluding other possible etiological factors related to BMS, we performed a complete blood count $(\mathrm{CBC})$ and patients with anemia were excluded. All patients signed an informed consent form before being included in the study. The diagnostic criteria for BMS included the following:

1) Burning sensation in all or some parts of the oral cavity with or without symptoms such as a change in taste for at least four months,

2) Normal oral mucosa without any lesions,

3) Absence of local or systemic factors producing similar symptoms.

The exclusion criteria consisted of known systemic diseases, age of less than 18 years, pregnancy, smoking habits, oral lesions and refusing to sign the informed consent form (9).

The subjects were divided to case and control groups using the block randomization technique.

All the subjects were examined before treatment and the burning sensation severity was evaluated based on numeric rating scale (NRS), with ' 0 ' indicating absence of burning sensation and '10' indicating the most severe burning sensation experienced by the subject. The subjects were asked to determine the severity of their burning sensation from 0 to 10 .

To prepare the medication, 220-mg zinc sulfate capsules were procured from the Alhavi company. The contents of half of the capsules were replaced with soluble pharmaceutical starch powder to prepare placebos. All capsules were then placed within coded glasses by a pharmacist and handed to the oral medicine specialist.

The patients in the case group were treated with zinc sulfate by the oral medicine specialist; 220-mg capsules of zinc sulfate (Alhavi Company, Iran) were administered twice daily and the replacement therapy was continued for three months. In the control group, placebo capsules, which looked the same as the zinc sulfate capsules, were administered two times daily for three months. After three months, burning sensation severity was evaluated and measured.

The serum zinc levels were determined and if these levels were not normal, treatment with zinc sulfate capsules continued for another month. After this period, the subjects were evaluated again and burning sensation severity was determined. All measurements were done by a physician blinded to the study procedures.

After the study, the control group patients also received zinc replacement therapy.

Data were analyzed with the SPSS 18 software. The Ttest was used to evaluate the means of burning sensation scores and serum zinc levels of the subjects. Statistical significance was set at $\mathrm{P}<0.05$.

\section{Results}

In the present study, 60 patients with BMS, who had zinc deficiency, were evaluated, with 30 subjects in the case and 30 in the control groups. In the case group, there were 28 females and two males; in the control group, there were 27 females and three males. The mean age of the subjects were $57.2 \pm 5.02$ and $55.6 \pm 7$ years in the case and control groups, respectively, with no statistically significant differences between the two groups $(P=0.06)$. The mean durations of affliction with BMS were $4.5 \pm 3.1$ and $4.1 \pm 1.5$ years in the case and control groups, respectively, with no statistically significant differences between the two groups $(\mathrm{P}=$ 0.4). The most common locations for burning sensation were the tongue, the entire oral cavity, gingiva and the buccal mucosa, in descending order.

Before intervention there was no difference in sensation severity and serum zinc level between the groups.

At the end of the study there was a significant difference in sensation severity and serum zinc level between the two groups (Table 1).

\section{Discussion}

The results of the present study showed that zinc supplementation decreases the severity of burning sensation in patients with BMS, although it does not alleviate it completely. 
Table 1. Severities of Burning Sensation and Serum Zinc Levels Before and After Treatment

\begin{tabular}{lccc}
\hline Variable & \multicolumn{2}{c}{ Treatment Group } & \multirow{2}{*}{ P Value } \\
\cline { 2 - 3 } & Case & Control & \\
\hline $\begin{array}{l}\text { Burning sensation before the } \\
\text { intervention }\end{array}$ & $5.6 \pm 1.3$ & $4.9 \pm 1.1$ & 0.05 \\
$\begin{array}{l}\text { Burning sensation after the } \\
\text { intervention }\end{array}$ & $1.4 \pm 0.9$ & $4.9 \pm 0.10$ & 0.001 \\
$\begin{array}{l}\text { Serum zinc level before the } \\
\text { intervention, } \mu \text { g/dL }\end{array}$ & $66 \pm 12.4$ & $61.8 \pm 10$ & 0.4 \\
$\begin{array}{l}\text { Serum zinc level after the } \\
\text { intervention, } \mu \mathrm{g} / \mathbf{d L}\end{array}$ & $82.4 \pm 11.6$ & $62.2 \pm 11$ & 0.009 \\
\hline
\end{tabular}

Zinc has an important role in the growth and development of the immune system and the neurologic functions. Zinc deficiency might affect the immune system and the repair processes of the mucosa and might have a role in BMS (3).

One study reported lower serum levels of zinc in patients with BMS compared to healthy subjects (10). Pekiner evaluated 30 BMS patients and 30 healthy subjects in 2009 and compared salivary levels of magnesium, zinc and copper in the subjects, reporting no significant differences between the two groups (2).

In a study by Cho et al. in 2010, twenty rats were divided to two groups of 10 . One group received a diet deficient in zinc and the other group received a standard diet. After 20 weeks, hyperkeratosis and an increase in mitotic activity were observed on the dorsum area of the tongue in rats receiving the Zn-deficient diet (3).

In another part of this study, $26 \%$ of subjects with BMS had zinc deficiency. These patients, who had no other laboratory abnormalities, received zinc supplementation. Relative recovery was reported in $71.9 \%$ of the subjects, consistent with the results of the present study (3).

It is not possible to compare the results of the present study with those of other studies from a clinical point of view due to the limited number of studies on BMS serum zinc levels.

\subsection{Conclusions}

Based on the results of the present study, it appears logical to evaluate the serum levels of zinc in patients with BMS and prescribe zinc supplementation in such patients.

\section{Footnote}

Authors' Contribution: Study concept and design: Fateme Arbabi-Kalati; analysis and interpretation of data: Fateme Arbabi-Kalati; drafting of the manuscript: Bahman Tahmtan; critical revision of the manuscript for important intellectual content: Fateme Arbabi-Kalati and Bahman Tahmtan.

\section{References}

1. Pezelj-Ribaric S, Kqiku L, Brumini G, Urek MM, Antonic R, Kuis D, et al. Proinflammatory cytokine levels in saliva in patients with burning mouth syndrome before and after treatment with low-level laser therapy. Lasers Med Sci. 2013;28(1):297-301. doi: 10.1007/s10103-012-1149-5. [PubMed: 22773117].

2. Pekiner FN, Gumru B, Demirel GY, Ozbayrak S. Burning mouth syndrome and saliva: detection of salivary trace elements and cytokines. J Oral Pathol Med. 2009;38(3):269-75. doi: 10.1111/j.16000714.2008.00734.x. [PubMed: 19141055].

3. Cho GS, Han MW, Lee B, Roh JL, Choi SH, Cho KJ, et al. Zinc deficiency may be a cause of burning mouth syndrome as zinc replacement therapy has therapeutic effects. J Oral Pathol Med. 2010;39(9):722-7. doi: 10.1111/j.1600-0714.2010.00914.x. [PubMed: 20618611].

4. Lamey PJ, Lamb AB. Prospective study of aetiological factors in burning mouth syndrome. Br Med J (Clin Res Ed). 1988;296(6631):1243-6. [PubMed: 3133028].

5. Gurvits GE, Tan A. Burning mouth syndrome. World J Gastroenterol. 2013;19(5):665-72. doi: 10.3748/wjg.v19.i5.665. [PubMed: 23429751].

6. Mock D, Chugh D. Burning mouth syndrome. Int J Oral Sci. 2010;2(1):14. doi: 10.4248/IJOS10008. [PubMed: 20690412].

7. Muzyka BC, De Rossi SS. A review of burning mouth syndrome. Cutis. 1999;64(1):29-35. [PubMed:10431669]

8. Sun A, Wu KM, Wang YP, Lin HP, Chen HM, Chiang CP. Burning mouth syndrome: a review and update. J Oral Pathol Med. 2013;42(9):649-55. doi: 10.1111/jop.12101. [PubMed: 23772971].

9. Arbabi-Kalati F, Bakhshani NM, Rasti M. Evaluation of the efficacy of low-level laser in improving the symptoms of burning mouth syndrome. J Clin Exp Dent. 2015;7(4):e524-7. doi: 10.4317/jced.52298. [PubMed: 26535101].

10. Maragou P, Ivanyi L. Serum zinc levels in patients with burning mouth syndrome. Oral Surg Oral Med Oral Pathol. 1991;71(4):447-50. [PubMed: 2052330]. 